\title{
A new transmucosal drug delivery system for patients with breakthrough cancer pain: the fentanyl effervescent buccal tablet
}

\author{
Enno Freye \\ Center of Ambulatory Pain Medicine, \\ Neuss-Uedesheim, Germany
}

Correspondence: Enno Freye

Deichstrasse 3, 4I468 Neuss-Uedesheim, Germany

Tel +49 2l3| 3|4242|

Email enno.freye@uni-duesseldorf.de

\begin{abstract}
Breakthrough pain, a transitory severe pain with the background of otherwise controlled persistent pain has a prevalence between $52 \%$ and $67 \%$ in outpatients with cancer. Medications for such sudden-onset pain require non-invasive delivery of a potent and short-acting opioid for rapid pain relief. Although oral transmucosal delivery of fentanyl citrate (OTFC) has been shown to provide better pain relief than a typical oral opioid administration such as morphine sulfate immediate release (MSIR) in the management of breakthrough pain in patients with cancer-related pain, newer delivery systems offer a potential for further enhancement of pain relief. The fentanyl effervescent buccal tablet (FBT) formulation employs a novel drug delivery system that relies on an effervescence reaction to improve buccal fentanyl absorption. Using the effervescence reaction results in the production and dissipation of carbon dioxide with a dynamic shift in $\mathrm{pH}$ as the tablet dissolves. The induced low $\mathrm{pH}$ favors dissolution of fentanyl citrate in saliva (higher water solubility). The subsequent increase in $\mathrm{pH}$ thereafter favors the buccal absorption of non-ionized fentanyl across the buccal mucosa. Such a $\mathrm{pH}$ "pumping" mechanism increases the permeation of fentanyl into and through the buccal to the vascular system from where the agent is transported to the specific opioid receptor sites in the CNS. Compared with OTFC, data in healthy volunteers show that the effervescence reaction employed in FBT increases the total amount and the speed of absorption of fentanyl being absorbed. Compared with OTFC there is an increase in peak fentanyl blood concentrations, and an enhancement of the amount of buccal delivery of fentanyl. Such favorable data are underlined by the results of clinical studies where the FBT technology was studied in patients with breakthrough pain in chronic malignant pathologies.
\end{abstract}

Keywords: breakthrough pain, fentanyl effervescent buccal tablet (FBT), cancer pain, effervescent technology

\section{Introduction}

Breakthrough pain is a transitory exacerbation of pain commonly associated with pain management in cancer patients. ${ }^{1,2}$ It has a prevalence between $52 \%$ and $67 \%$ in otherwise well-controlled, persistent cancer-related pain, ${ }^{3,4}$ and up to $89 \%$ in palliative cancer units. ${ }^{5}$ It is often induced by weight bearing/excessive movement, during defecation/urination, coughing, while eating or even spontaneously without any known cause, sometimes reflecting an end-of-dose failure. ${ }^{4,6,7}$ Although breakthrough pain is heterogeneous, with a somatic, visceral or neuropathic origin, it is usually similar in etiology and pathophysiology to persistent pain showing a moderate to severe intensity. Because of all these features breakthrough pain demands immediate and adequate relief.

With a mean frequency of 1 to 4 episodes/day, a mean time to peak effect of 3 to 10 minutes and a mean duration of 15 to 60 minutes, ${ }^{1,8}$ such episodic increase in pain intensity demands a fast-acting therapeutic regimen. Although relief from breakthrough pain can be derived from general measures, such as changing position, hot and 
cold compresses, massage, counseling and/or non-analgesics, such practices provide modest relief at best. ${ }^{9}, 10$ Therefore the current approach in the management of patients with chronic cancer pain and breakthrough pain involves a medication on top of the basic opioid, with a rapid onset, and a short duration of action, permitting titration to effect. In addition, it should induce only minimal side effects, and should be applied by the patient by mouth..$^{5,11}$ Because the oral route is the most convenient mode of application, a variety fastacting opioids have been developed, all of which, however, demonstrate a mismatch between the temporal characteristics of breakthrough pain and the onset of analgesia associated with standard, short-acting, orally administered opioids. Conventionally treated with a supplemental pro re nata (PRN) dose of a fast-release tablet or oral solution of morphine, the agents have a low lipophilic profile, which results in a slow permeation through the blood-brain barrier taking up to 60 minutes till maximum efficacy. ${ }^{12}$ Although a novel formulation of morphine with an effervescent delivery system (Painbreak ${ }^{\circledR}$, Riemser Company, Germany) displays a faster onset of action than the immediate-release formulation, ${ }^{13}$ oral transmucosal fentanyl citrate (OTFC) has become a mainstay in the treatment of breakthrough pain, ${ }^{14}$ because it provides faster absorption of the lipophilic fentanyl than any other oral formulation. ${ }^{9}$ However, there are limitations of this formulation including conveniency in an outpatient setting as the fentanyl stick does require a handle for swabbing along the buccal surface, a dental decay because of its sugar content, and a time span to become effective that still is far from being optimal. ${ }^{8,15,16}$
Therefore, a new formulation of fentanyl, the fentanyl buccal tablet (FBT), was designed to provide an even faster onset of analgesia. The buccal tablet has been approved by the FDA and has been marketed in the US since 2006 under the brand name Fentora ${ }^{\circledR}$ (Cephalon, Inc., Salt Lake City, Utah, USA) for the treatment of breakthrough pain in opioid-tolerant cancer patients using standard regularrelease opiates. In April 2008 the EMEA approved FBT in Europe for the same indication, under the brand name Effentora $^{\mathrm{TM}}$. Although OTFC is effective in this indication because of its rapid onset of action, the newly approved formulation offers certain advantages. Further refinements of the fentanyl formulation were made in order to increase patient compliance, while at the same time increasing transmucosal absorption of fentanyl across the buccal mucosa, resulting in a further shortening in onset of action and an increase of the amount of the opioid being absorbed into the bloodstream. ${ }^{17}$ In addition, the inconspicuous use of the buccal tablet by patients in public when there is an incidence of breakthrough pain results in a higher comfort.

FBT displays a further increase in onset of action over the OTFC solid drug-matrix formulation. In order to achieve these advantages practical considerations that affect the microenvironment adjacent to the dissolving of FBT need to be borne in mind. Patients are instructed to place the buccal tablet above a molar tooth between the upper cheek and allow it to dissolve for approximately 14 to 25 minutes (Figure 1). FBT has a mildly bitter taste, and if the tip of the tongue is placed on a dissolving tablet, a slow "fizzing" sensation similar to that produced by a carbonated beverage

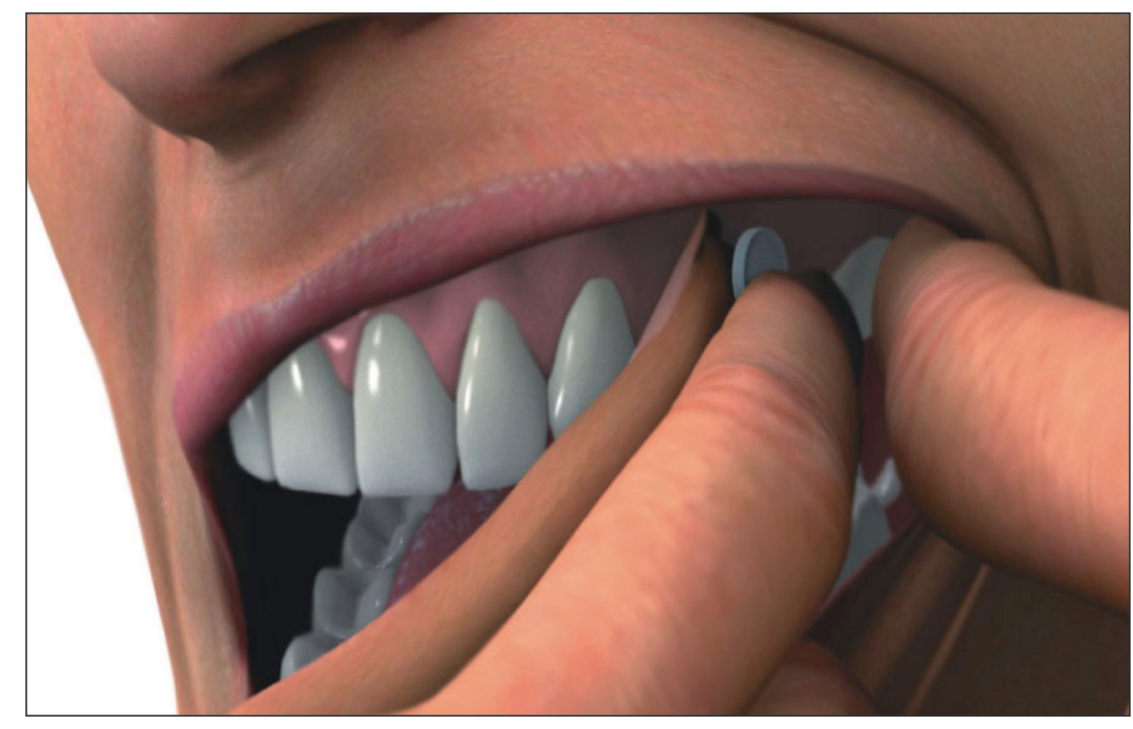

Figure I The principle of placing a fentanyl effervescent buccal tablet in the cheek pouch. 
may be experienced. After 30 minutes, if remnants from the buccal tablet remain, they may be swallowed with a glass of water. The buccal tablet should not be sucked, chewed or swallowed, as this will result in lower plasma concentrations than when taken as directed.

\section{Formulation of the FBT for breakthrough cancer pain}

Similar to the oral morphine effervescent formulation Painbreak $^{\circledR}$, effervescence can be of use as a penetration enhancer. ${ }^{18}$ The basic principles of action of an effervescent formulation are to

- open tight junctions of the mucosal barrier,

- increase the hydrophilic nature of the solvent by an enhanced penetration through the water layer of the buccal membrane

- increase the fluid volume with a drag effect through the mucous membrane.

In principal, by adding citric acid and sodium bicarbonate to the lipophilic opioid fentanyl, and making contact with saliva, carbon dioxide is released, which dissolves to form carbonic acid, ensuring a decrease of $\mathrm{pH}$ and an increase in water solubility of fentanyl. This process decreases preexisting ions (cations as well as anions), which are now forced to form molecules, being neutral on the outside. Since passive diffusion of the active ingredient fentanyl increases proportionally with the higher amount of uncharged molecules, higher amounts of fentanyl will penetrate through the unstirred water layer of the mucous membrane.

Proof for the above hypothesis has been demonstrated in clinical studies using this Oravescent ${ }^{\circledR}$ Technology (CIMA Labs, Inc., Eden Prairie, MN, USA). There was an intial faster rise in serum levels and a more complete absorption of fentanyl, resulting in a quicker onset of action, which is of advantage in breakthrough cancer pain. ${ }^{19}$ In this study kinetics of FBT demonstrated proportionality of dosages ranging from $200,500,810$ to $1080 \mu \mathrm{g}$ in healthy human volunteers, with a faster onset and higher maximum serum fentanyl concentration compared with a similar dose to the conventional transmucosal fentanyl citrate (Figure 2).

\section{Pharmacokinetic parameters of fentanyl effervescent buccal tablets in adults}

After being absorbed into the blood stream, and contrast to morphine, fentanyl enters the central nervous system (CNS) much faster because it rapidly crosses the blood-brain barrier once it enters the systemic circulation. ${ }^{20}$ The rate constant

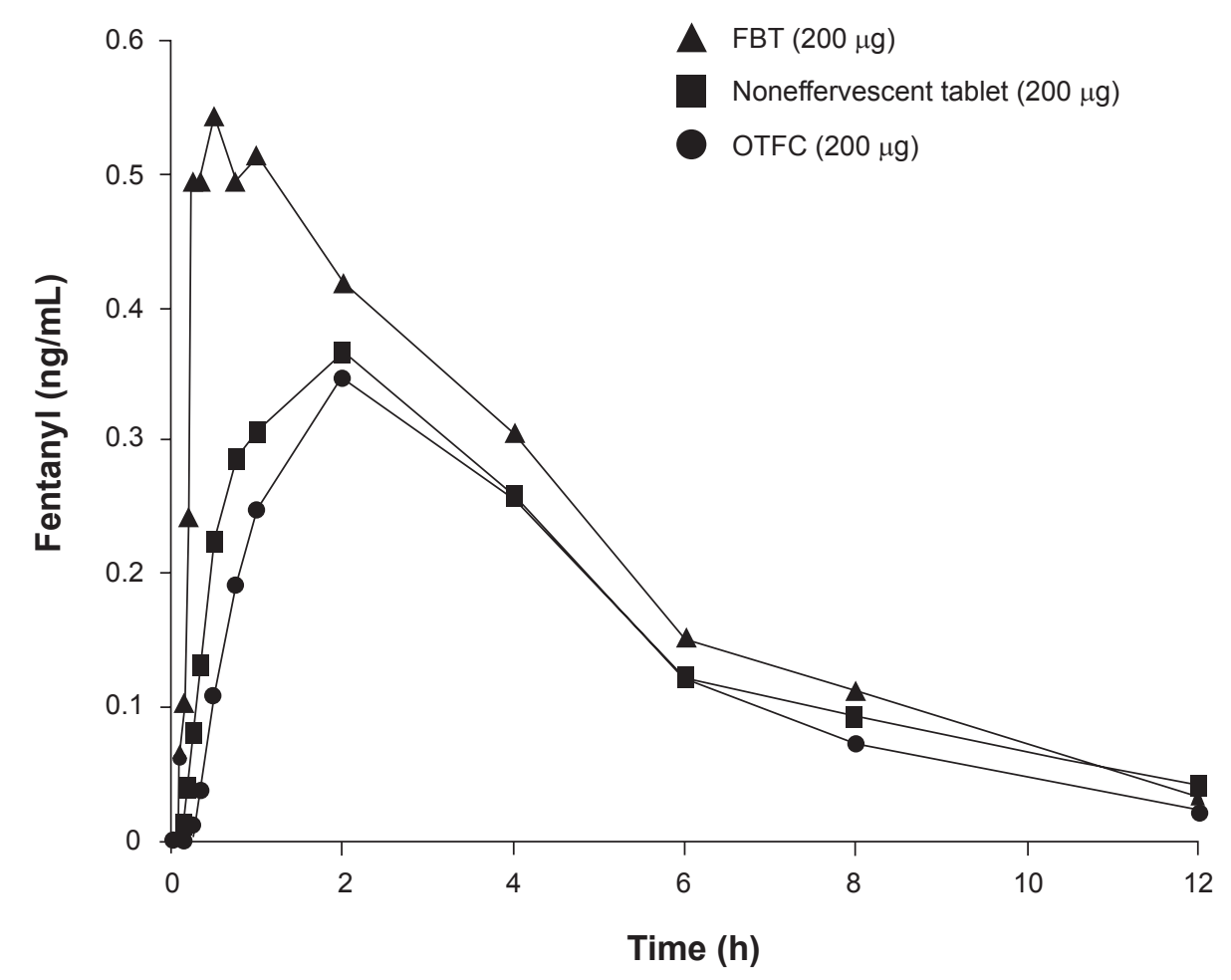

Figure 2 Serum fentanyl concentrations after administration of fentanyl effervescent buccal tablets (FBT) $200 \mu \mathrm{g}$, fentanyl $200 \mu \mathrm{g}$ tablets without effervescent agents, compared with oral transmucosal fentanyl citrate (OTFC) $200 \mu \mathrm{g}$. Adapted from. ${ }^{19}$ 
for the equilibrium of fentanyl between the blood and CNS is 6 minutes, ${ }^{20}$ whereas the same rate constant for morphine is approximately 17 minutes. ${ }^{21}$ The novel formulation of FBT therefore results in a pharmacokinetic profile that is expected to provide greater clinical benefits in breakthrough cancer pain than OTFC. For example, in clinical studies FBT is rapidly absorbed with a $\mathrm{T}_{\max }$ - an indicator of the time to maximum analgesia - of 46 minutes (Figure 3, Table 1), in contrast to OTFC, which has a $\mathrm{T}_{\max }$ of 91 minutes. Mean serum concentrations show a biexponential decline after the peak levels after FBT at a dose $400 \mu \mathrm{g} .{ }^{17}$ However, individual profiles for approximately half of the subjects in these studies show a triexponential decline, which is consistent with the known pharmacokinetic profile of fentanyl. Then the agent is rapidly distributed from the serum to highly perfused tissues such as the brain, heart and lungs, and then redistributed back to the plasma. ${ }^{17}$ It is the rapid distribution to the brain that is presumed to account for the fast onset of action of a fentanyl-induced analgesia.

The initial distribution half-life to the lower perfused tissues is followed by an elimination/gastrointestinal absorption phase and a slower phase of distribution to the deep tissue compartments. This tissue distribution, combined with biotransformation in the liver by the CYP3A4 enzyme system, results in the formation of inactive norfentanyl, and an appropriate decline in plasma concentrations below clinically relevant threshold levels. ${ }^{22}$ Fentanyl has been chosen for transmucosal delivery in the FBT formulation because it has a high bioavailability of $65 \%( \pm 20 \%$ SD) combined with a high fraction being absorbed via the mucous membranes of $48 \%( \pm 31.8 \%$ SD $) .{ }^{23}$ Additional studies demonstrate that the plasma $\mathrm{C}_{\max }$ of FBT increases in a dose-proportional manner over a dose range of 100 to $800 \mu \mathrm{g}$ (Table 1).

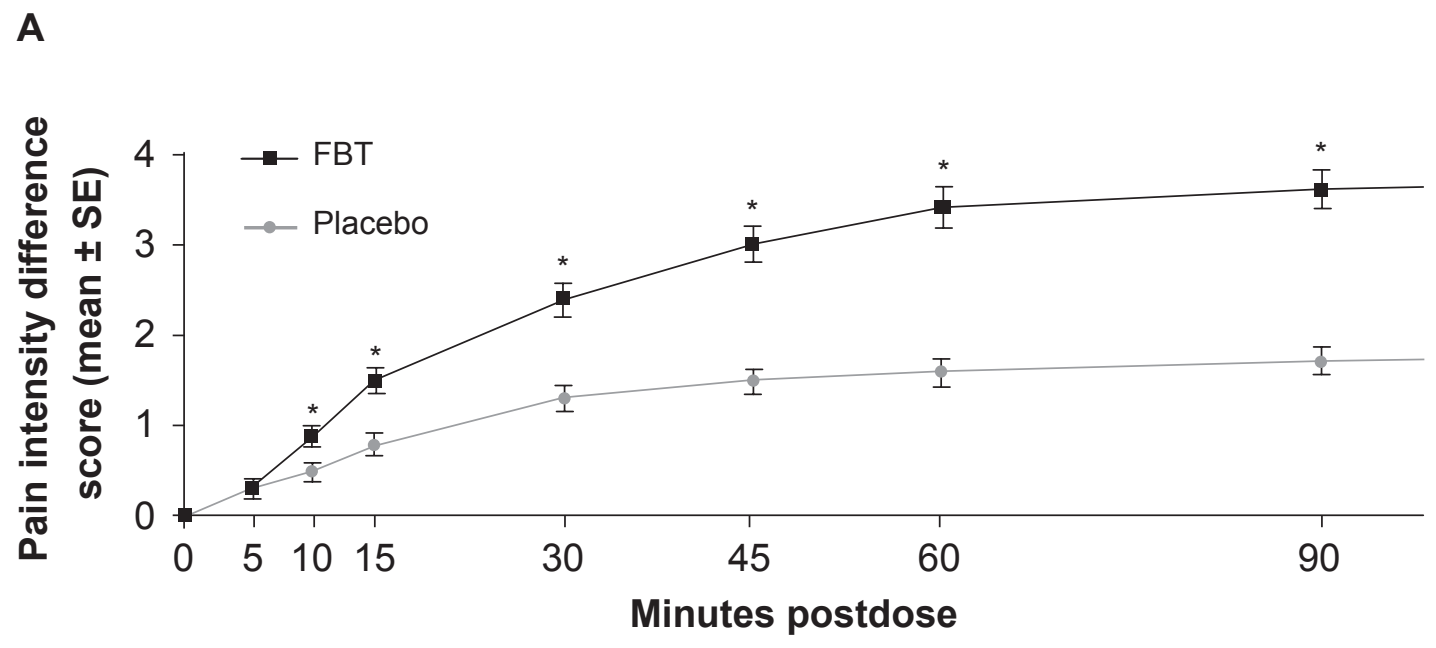

B

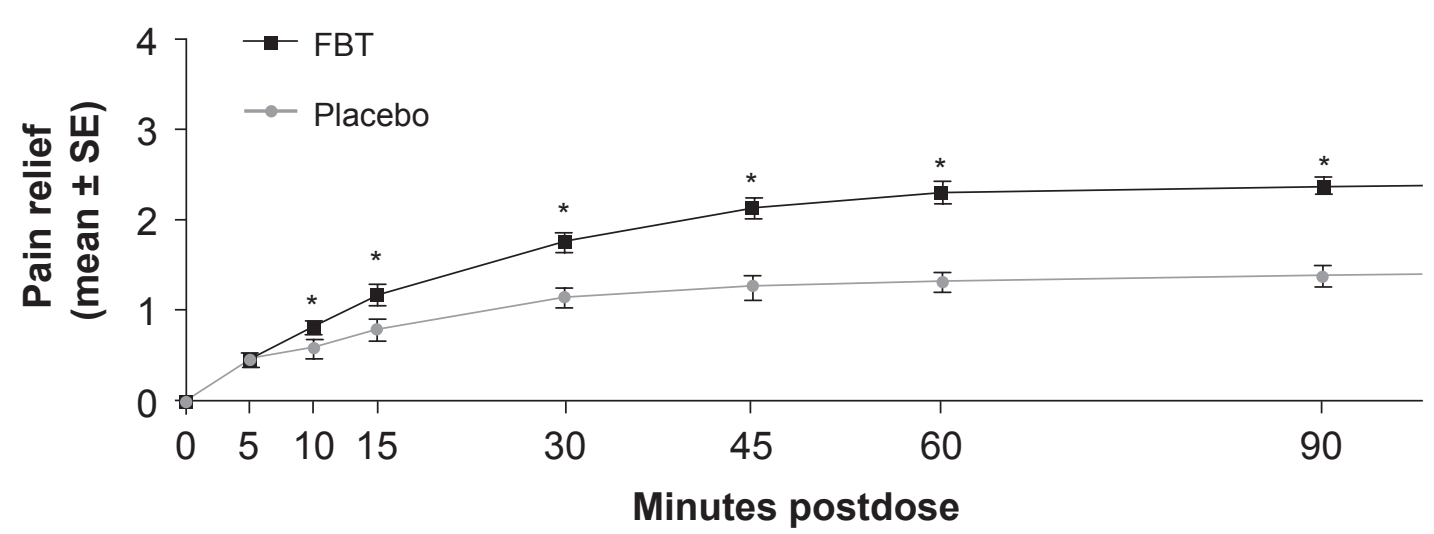

Figure 3 Effect of fentanyl buccal tablet (FBT) and placebo on: A. Pain intensity difference and B. Pain relief in opioid-tolerant patients with breakthrough pain associated with chronic cancer pain. Adapted from. ${ }^{31}$ 
Table I Pharmacokinetic properties of different single doses of fentanyl effervescent buccal tablets

\begin{tabular}{|c|c|c|c|c|c|}
\hline $\begin{array}{l}\text { Dose } \\
(\mu g)\end{array}$ & $\begin{array}{l}T_{\max } \mathbf{h} \\
\text { (median, 90\% Cl) }\end{array}$ & $\begin{array}{l}C_{\max } n g / m L \\
(m e a n \pm S D)\end{array}$ & $\begin{array}{l}t_{1 / 2} h \\
\text { (median, 90\% Cl) }\end{array}$ & $\begin{array}{l}\text { AUC } \\
0->T_{\max }(n g * h / m L)\end{array}$ & $\begin{array}{l}\text { AUC } \\
0->\infty(n g * h / m L)\end{array}$ \\
\hline 100 & $0.75(0.4-3.02)$ & $0.25(0.14)$ & $2.63(1.5-13.6)$ & $0.80(0.26)$ & $0.98(0.37)$ \\
\hline 200 & $0.67(0.33-3.0)$ & $0.40(0.18)$ & $4.43(1.9-20.8)$ & $1.39(0.46)$ & $2.11(1.13)$ \\
\hline 400 & $0.58(0.33-3.0)$ & $0.97(0.53)$ & II.I (3.4-20.6) & $2.90(0.92)$ & 4.72 (1.95) \\
\hline 800 & $0.67(0.4-3.0)$ & $1.59(0.90)$ & II.7 (4.6-28.6) & $5.27(1.85)$ & 9.05 (3.72) \\
\hline
\end{tabular}

Adapted from. ${ }^{24}$

Abbreviations: $\mathrm{Cl}$, confidence interval; $\mathrm{SD}$, standard deviation; $\mathrm{AUC}$, area under the curve.

At doses between 100 to $800 \mu \mathrm{g}$ of FBT the area under the curve (AUC $0->t$ ) and $\mathrm{C}_{\text {max }}$ show a linear proportional relationship to the administered dose, with a median time to maximum concentration $\left(\mathrm{T}_{\max }\right)$ in the range of 35 to 45 minutes. In addition, a single-dose study of 270 to $1300 \mu \mathrm{g}$ for dose proportionality found an overall dose effect, and for dosages greater than $810 \mu \mathrm{g}$ showed a less-than-dose-proportional increase in $\mathrm{C}_{\max }{ }^{25}$ Such effects might be attributed to saturation at the site of administration at the buccal mucosa, because administration of four $100 \mu \mathrm{g}$ tablets in contrast to one single $400 \mu \mathrm{g}$ tablet produces slightly higher AUC values. Such differences might be due to the higher surface area of the tablet coming into contact with the buccal mucosa. ${ }^{23}$

Two pharmacokinetic studies compared the different profiles of FBT and OTFC. FBT demonstrated a significant faster rate $\left(\mathrm{T}_{\max }\right)$ and a significant greater extent $\left(\mathrm{C}_{\max }\right.$ and AUC $0->\infty$ ) of fentanyl movement into the systemic circulation. ${ }^{25}$ Although the $\mathrm{T}_{\max }$ of the effervescent fentanyl delivery system is longer than onset-to-pain-intensity peak time of breakthrough pain, the new formulation has certain advantages. With a median $\mathrm{T}_{\text {max }}$ of 47 versus 91 minutes and a higher bioavailability of $65 \%$ versus $47 \%$, such data favor FBT. ${ }^{26}$ Together with these favorable pharmacokinetic data and assuming that opioid blood concentrations are closely related to clinical effect, ${ }^{27} \mathrm{FBT}$ allows a more straightforward titration of the opioid fentanyl to achieve the desired analgesic level. Although the observation that multiple dosing with FBT shows predictable pharmacokinetics, which also contributes to the ease of dose titration, ${ }^{17}$ a faster clinical effect, based on a shorter $T_{\max }$, can only be assumed. Further studies are therefore needed to substantiate such an effect.

The variation in the time taken for FBT to dissolve in the buccal pouch - also called the 'dwell time' - has led to the question of whether such variability affects absorption of FBT, which may be of relevance to subsequent plasma levels. ${ }^{28}$ Post-hoc analysis of the pharmacokinetics of FBT in healthy volunteers indicates that the rate and extent of fentanyl absorption are not affected by the time taken for complete dissolution in the buccal cavity. ${ }^{28}$ Such findings suggest that any residue left in the buccal cavity is inactive and that variability in dwell time is not likely to be important for achieving the desired analgesic effect. Therefore, patients are instructed to swallow with water any of the buccal tablet remaining after 30 minutes. Although the primary pharmacokinetic studies with fentanyl buccal tablets have been carried out in healthy volunteers, other studies involving cancer patients with $(n=8)$ or without $(n=8)$ clinical and functional grades of mild mucositis (grade 1) revealed that the absorption profile of FBT $200 \mu \mathrm{g}$ was similar in both groups. ${ }^{29}$ The results indicate that FBT can also be considered in opioidtolerant patients who also exhibit mild mucositis.

\section{Clinical efficacy of oral FBT in patients with breakthrough pain}

The clinical efficacy of FBT in treating breakthrough pain associated with cancer pain has been well established in two randomized, placebo-controlled trials in opioid-tolerant cancer patients. The two studies, one with 123 patients $^{30}$ the other with 125 patients ${ }^{31}$ received FBT in a double-blind fashion. Both documented clinically significant improvement in pain scores versus placebo (Figure 3). The significant greater reduction ( $p<0.05$ and $p<0.0001$, respectively) in pain intensity was achieved after FBT administration compared with placebo as early as 15 and 10 minutes, respectively. Interestingly, patients with moderate or severe pain at baseline generally experienced greater improvements in pain than those with mild pain.

In these studies, for $35 \%$ of FBT-treated episodes, the efficacy at 60 minutes was rated as 'very good' or 'excellent'. Also, use of supplemental medication for pain relief occurred more frequently during episodes of breakthrough pain when patients received placebo (50\%) than with FBT $(23 \%)$. In these studies the beneficial effect of an effervescent 
fentanyl delivery system to a clinical endpoint of pain relief was compared only with placebo. Thus, there is the need to demonstrate its effect in further studies, comparing the clinical benefits with other compounds.

In the studies with FBT as well as in other studies where breakthrough pain was treated with an orally fast-acting morphine tablet, ${ }^{32}$ an effervescent solution ${ }^{13}$ or with OTFC, ${ }^{33,34}$ there was no simple linear relationship between the effective dose and the dose of the background opioid regimen. Not only does such an approach lack a solid base of evidence, but also accumulating data suggest that the optimal dose of breakthrough pain medication may bear little relationship to the around-the-clock dose. ${ }^{31}$ Such results underline the need to titrate individually to effectiveness rather than calculate the percentage of an existing opioid regimen as previously suggested. ${ }^{33,35,36}$

\section{Safety and tolerance of the FBT}

In both reported placebo-controlled studies, the most commonly observed adverse events were typical of those observed with other opioid medications, with the exception of application-site reactions (Table 2).

The obvious difference in side effects between the studies of Slatkin and of Portenoy may well be attributed to the difference in around-the-clock and supplemental medication. For instance, in Portenoy's study ${ }^{37}$ most patients used either a fentanyl transdermal formulation (28\%), morphine (34\%) or oxycodone $(36 \%)$ extended release as their basic opioid medication. Most patients in the Slatkin study ${ }^{31}$ used either oxycodone, an oxycodone-acetaminophen combination $(54 \%)$, or the fentanyl patch $(32 \%)$ while only $20 \%$ took morphine as their basic medication. The well established fact that morphine alone results in a higher percentages

Table 2 Adverse events occurring in $\geq 5 \%$ of patients in the placebo-controlled trials of fentanyl buccal tablet in opioid-tolerant cancer patients experiencing breakthrough pain

\begin{tabular}{lll}
\hline Adverse events (\%) & Study $\mathbf{n}=\mathbf{I} \mathbf{2 3}$ & Study $\mathbf{n}=\mathbf{I} \mathbf{2 5}$ \\
\hline Nausea & $27(22)$ & $16(13)$ \\
Dizziness & $27(22)$ & $14(1 \mathrm{I})$ \\
Headache & $18(15)$ & $8(6)$ \\
Fatigue & $15(12)$ & $10(8)$ \\
Constipation & $10(8)$ & $7(6)$ \\
Vomiting & $13(1 \mathrm{I})$ & $8(6)$ \\
Somnolence & $12(10)$ & $\mathrm{NR}$ \\
Asthenia & $9(7)$ & $\mathrm{NR}$ \\
\hline
\end{tabular}

After ${ }^{30,31}$

Abbreviation: NR, not reported. of side effects than other opioids, especially in an elderly population, ${ }^{38,39}$ may account for the difference in the percentage of observed effects.

Another potential serious side effect is respiratory depression. No data on serious adverse events from respiratory depression and/or chest wall rigidity were observed in clinical trials, nor have they been found in the available literature in patients following correct dosing of FBT. ${ }^{30,31,40}$

\section{Switching from OTFC to FBT - dosing recommendations}

The important consequence of the novel formulation of the FBT is that the bioavailability of fentanyl is higher than with an equivalent dose of OTFC. ${ }^{23}$ Such a difference has to be taken into consideration when wanting to switch from OTFC to FBT for breakthrough pain episodes. In a crossover study in healthy volunteers, an $\sim 30 \%$ smaller dose of FBT achieved systemic exposure comparable to OTFC, although significant individual variation was observed. ${ }^{23}$ Such finding should be taken into consideration when switching patients between these two formulations in order to prevent patients from being subjected to inappropriately high or low systemic levels of fentanyl. Because FBT is available in tablets strengths of 100, 200, 400, 600 and $800 \mu \mathrm{g}$, and taking into account a $30 \%$ smaller dose of FBT than OTFC, switching therefore can be tailored to the patient's individual requirements. This, however, is followed by an up or down dose titration to provide effective analgesia while avoiding adverse side effects.

The initial dose of FBT in breakthrough cancer pain is $100 \mu \mathrm{g}$. Titration should start with one $100 \mu \mathrm{g}$ tablet and should subsequently be performed with the $200 \mu \mathrm{g}$ tablet and multiples of this tablet strength, ie, the patient should use two $200 \mu \mathrm{g}$ tablets with one on each side of the mouth in the buccal cavity or three $200 \mu \mathrm{g}$ tablets with one on one side of the mouth in the buccal cavity and two in the other side or two $200 \mu \mathrm{g}$ tablets on each side of the mouth in the buccal cavity totaling four $200 \mu \mathrm{g}$ tablets. Using more than 4 tablets simultaneously has not been studied and is therefore not advised. In addition, it is important to minimize the number of strengths available to patients at any time to prevent confusion and possible overdose. Once a successful dose has been established during the titration, patients should continue to take their dose as a single tablet of that given strength and must wait 4 hours before treating a new breakthrough pain episode. If more than 4 breakthrough cancer pain episodes occur per day, a dose readjustment of the maintenance opioid used for the persistent pain should be re-evaluated and 
possibly increased. Based on the dosing recommendation and safety information, prior feedback with the pain specialist is required. Before prescribing the formulation for a longer period of time (eg, up to 6 months with a quantity limit of 4 units per day), the pain specialist should ensure that there is no drug diversion and/or illicit use of the agent. ${ }^{41}$

\section{Safety precautions when using the FBT}

Recently, the FDA issued a Public Health Advisory and a Healthcare Professional Sheet to alert healthcare professionals and consumers of potential safety issues with FBT after reports of isolated cases of deaths and other adverse events. ${ }^{42}$ The reported deaths all occurred as a result of improper patient selection, eg, use in opioid non-tolerant patients, improper dosing, and/or improper product substitution. Therefore, it is critical to follow product labeling when administering FBT. The tablet should not be used in patients who are not opioid tolerant and it is dangerous to use FBT for any short-term pain such as headache or migraine. Furthermore, physicians must be aware that FBT cannot be substituted for OTFC on a microgram base because, as demonstrated, FBT delivers more fentanyl to the blood than OTFC and, thus, dose-for-dose substitution can result in a fatal overdose. Therefore the following key points underline the need for appropriate patient selection, and proper dosing and administration of FBT in order to reduce the risk of respiratory depression.

In addition to carefully following dosing instructions and patient selection, the following criteria are to be observed in order to avoid potential respiratory depression (10 rules in breakthrough pain):

1. FBT at any dosage should not be used in opioid nontolerant patients, ie, patients who are not taking aroundthe-clock opioids.

2. FBT should be used only for the labeled indication.

3. FTB should not be prescribed for patients with acute pain, postoperative pain, headache/migraine, or sports injuries, even if they are deemed suitable recipients of other opioids on a PRN basis.

4. Since FTB is not a generic version of OTFC, the buccal tablet should not be substituted for OTFC or any other fentanyl-containing products.

5. For unrelieved breakthrough cancer pain, patients should not take more than 2 FBT tablets per breakthrough pain episode, except during dose titration.

6. Patients must wait at least 4 hours before treating another breakthrough pain episode with FBT.
7. In cases of more than 4 cancer pain breakthrough episodes per day, a readjustment of the maintenance opioid dose should be considered.

8. FBT contains a highly potent opioid, which has the potential for respiratory depression especially when used in opioid-naïve patients. Respiratory depression can rapidly be reversed by the specific antagonist naloxone. When doing so, the dose of the antagonist should be titrated to effect in order to retain sufficient analgesia. ${ }^{43}$

9. Usually opioids are effective in treating breakthrough pain. In certain situations, however, (eg, neuropathic pain) these agents are not sufficient to alleviate nociception. Then the non-specific NMDA antagonist ketamine (preferably as a nasal spray) presents an alternative solution. ${ }^{44}$

10. Finally, FBT is a schedule II agent, which has the potential for abuse, addiction and diversion when used in patients without cancer pain.

\section{Conclusion}

From a clinical perspective, FBT offers a number of advantages over older agents used in breakthrough cancer pain. Compared with standard regular-release opiates, such as oxycodone or morphine, it has to be expected that the peak analgesic effect will occur with FBT before the other agents produce any significant analgesic effect. Because FBT enhances bioavailability of fentanyl across the buccal mucosa, it is also the preferred rapid-onset opiate when a patient has difficulty swallowing and/or in situations where there is poor gastrointestinal absorption. Thus, drugs like FBT represent a new technology, which optimizes the ability to rapidly control breakthrough cancer pain and improve pain control, thereby improving quality of life. In spite of such recent advancement in the treatment of breakthrough pain, even when using FBT, challenges will remain as there is still scope for further optimization.

\section{Disclosures}

The author has no conflicts of interest to declare.

\section{References}

1. Portenoy RK, Bennett DS, Rauck R, et al. Prevalence and characteristics of breakthrough pain in opioid-treated patients with chronic noncancer pain. J Pain. 2006;7:583-591.

2. Caraceni A, Martin iC, Zecca E, Portenoy RK; Working Group of an IASP Task Force on Cancer Pain. Breakthrough pain characteristics and syndromes in patients with cancer pain: an international survey. Palliat Med. 2004;18:177-183.

3. Portenoy RK, Hagen NA. Breakthrough pain: definition, prevelance and characteristics. Pain. 1990;41:273-281.

4. Portenoy RK, Payne D, Jacobsen P. Breakthrough pain: characteristics and impact in patients with cancer pain. Pain. 1999;81:129-134. 
5. Zappetella G. Opioids for cancer breatkthrough pain: a pilot study reporting patient assessment of time to meaningful pain relief. J Pain Symptom Manage. 2008;35:563-567.

6. Fine PG, Busch MA. Characteristics of breakthrough pain by hospice patients and their caregivers. J Pain Symptom Manage. 1998;16:179-183.

7. Simmonds MA. Management of breakthrough pain due to cancer. Oncology. 1999;13:1103-1114.

8. Bennett DS, Burton A, Fishman S, et al. Consensus panel recommendations for the assessment and management of breakthrough pain. Part 1. Assessment. Pharm Ther. 2005;30:296-301.

9. Mercadante S, Arcuri E. Breakthrough pain in cancer patients: pathophysiology and treatment. Cancer Treat Rev. 1998;24:425-432.

10. Fine PG. Breakthrough cancer pain: epidemiology, characteristics and management. CNS Drugs. 2000;13:313-319.

11. William L. Management of breakthrough pain in patients with cancer. Drugs. 2008;68:913-924.

12. Cube von B, Teschemacher HJ, Herz A, Hess R. Permeation of morphine-like substances to their site of antinociceptive action in the brain after intravenous and intraventricular application and dependence on lipid solubility. Arch Pharmacol. 1970;265:455-502.

13. Freye E, Levy JV, Braun D. Effervescent morphine results in faster relief of breakthrough pain in patients compared with immediate release morphine sulfate tablet. Pain Pract. 2007;7:324-331.

14. Coluzzi PH, Shwartzberg L, Conroy Jr JD, Charapata S, Gay M, Busch MA, et al. Breakthrough cancer pain: a randomized trial comparing oral transmucosal fentanyl citrate $\left(\mathrm{OTFC}^{\circledR}\right)$ and morphine sulfate immediate release $\left(\mathrm{MSIR}^{\circledR}\right)$. Pain. 2001;91:123-130.

15. Mercadante S, Radbruch L, Caraceni A, Steering Committee of the European Association for Palliative Care (EAPC). Episodic (breakthrough) pain: consensus conference of an expert working group of the EAPC. Cancer. 2002;94:832-839.

16. Hwang SS, Chang VT, Kasimis B. Cancer breakthrough pain characteristics and responses to treatment at a VA medical center. Pain. 2003;101:55-64.

17. Darwish M, Tempero K, Kirby M, Thompson J. Pharmacokinetics and dose proportionality of fentanyl effervescent buccal tablets in healthy volunteers. Clin Pharmacokinet. 2005;44:1279-1286.

18. Eichmann JD, Robinson JR. Mechanistic studies on effervescentinduced permeability enhancement. Pharm Res. 1998;15:925-930.

19. Pather SI, Siebert JM, Hontz J. Enhanced buccal delivery of fentanyl using the OraVescent drug delivery system. Drug Delivery Technol. 2001;1:54-57.

20. Scott JC, Ponganis KV, Stanski DR. EEG quantification of narcotic effect: The comparative pharmacodynamics of fentanyl and alfentanil. Anesthesiology. 1985;62:234-241.

21. Kramer TH, d'Amours RH, Buettner C. Pharmacodynamic model of the effects of morphine-6-glucuronide during patient controlled analgesia. Clin Pharmacol Ther. 1996;59:132.

22. Mather LE. Clinical pharmacokinetics of fentanyl and its newer derivatives. Clin Pharmacokinet. 1983;8:422-446.

23. Darwish M, Kirby M, Robertson P, Tracewell W, Jiang JG. Absolute and relative bioavailability of fentanyl buccal tablet and oral transmucosal fentanyl citrate. J Clin Pharmacol. 2007;47:343-350.

24. Darwish M, Kirby M, Robertson PJ, Tracewell W, Jiang JG. Pharmacokinetic properties of fentanyl effervescent buccal tablets: a phase I, open-label, crossover study of single-dose 100, 200, 400, and $800 \mu \mathrm{g}$ in healthy adult volunteers. Clin Ther. 2006;28:707-714.

25. Darwish M, Tempero K, Kirby M, Thompson J. Relative bioavailability of the fentanyl effervescent buccal tablet (FEBT) 1080 pg versus oral transmucosal fentanyl citrate $1600 \mathrm{pg}$ and the dose proportionality of FEBT 270 to $1300 \mu \mathrm{g}$ : a single-dose, randomized, open-label, threeperiod study in healthy adult volunteers. Clin Ther. 2006;28:715-724.
26. Darwish M, Kirby M, Robertson PJ, Hellriegel E, Jiang JG. Comparison of equivalent doses of fentanyl buccal tablets and arteriovenous differences in fentanyl pharmacokinetics. Clin Pharmacokinet. 2006; $45: 843-850$.

27. Hill HF, Chapman CR, Saeger LS, Bjurtsrom R, Walter MH, Schoenen RB, et al. Steady-state infusions of opioids in humans. II. Concentration-effect relationships and therapeutic margins. Pain. 1990;43:69-79.

28. Darwish M, Kirby M, Jiang JG. Effect of buccal dwell time on the pharmacokinetic profile of fentanyl buccal tablet. Expert Opin Pharmacother. 2007;8:2011-2016.

29. Darwish M, Kirby M, Robertson P, Tracewell W, Jiang JG. Absorption of fentanyl from fentanyl buccal tablet (FBT) in cancer patients with or without oral mucositis - a pilot study. Clin Drug Investig. 2007;27:605-611.

30. Portenoy RK, Taylor D, Messina J, Tremmel L. A randomized, placebocontrolled study of fentanyl buccal tablet for breakthrough pain in opioid-treated patients with cancer. Clin J Pain. 2006;22:805-811.

31. Slatkin NE, Xie F, Segal TJ. Fentanyl buccal tablet for relief of breakthrough pain in opioid-tolerant patients with cancer-related chronic pain. J Spupport Oncol. 2007;5:327-334.

32. Fallon MT, Hanks GW. Morphine, constipation and performance status in advanced cancer patients. Palliat Med. 1999;13:159-1560.

33. Jacox A, Carr DB, Payne R. New clinical-practice guidelines for the management of pain in patients with cancer. $N$ Engl $J$ Med. 1994;330:651-655.

34. Hanks GW, Nugent M, Higgs CMB, Bush MA. Oral transmucosal fentanyl citrate in the management of breakthrough pain in cancer: an open, multicentre, dose-titration and long-term use study. Pall Med. 2004; 18:698-704.

35. Ventafridda V, Tamburini M, Caraceni A, De Conno F, Naldi F. A validation study of the WHO method of cancer pain relief. Cancer. 1987:59:851-856.

36. Hanks GW, de Conno F, Cherny N, Hanna, Kalso E, McQuay HJ. Morphine and alternative opioids in cancer pain: the EAPC recommendations. Br J Cancer. 2001;84:587-593.

37. Portenoy RK, Messina J, Xie F, Peppin J. Fentanyl buccal tablet (FBT) for relief of breakthrough pain in opioid-treated patients with chronic low back pain: a randomized, placebo-controlled study. Curr Med Res Opin. 2007;23:223-233.

38. Weiss S, Emanuel L, Fairclough D, Emanuel E. Understanding the experience of pain in terminally ill patients. Lancet. 2001;357:1311-1315.

39. Ahmedzai S, Brooks D. Transdermal fentanyl versus sustained-release oral morphine in cancer pain: preference, efficacy, and quality of life. J Pain Symptom Manage. 1997;13:254-261.

40. Webster LR. Fentanyl buccal tablets. Expert Opin Investig Drugs. 2006; 15:1469-1473.

41. Freye E. Detection of illicit use of opioids in primary care. In: Freye E, Levy J (eds). Opioids in Medicine - A Comprehensive Review on the Mode of Action and the Use of Analgesics in Different Clinical Pain States. Dordrecht/NL: Springer Science + Business Media BV; 2008. p. $412-465$.

42. Food and Drug Administration. FDA Warns of Potential Serious Side Effects with Breakthrough Cancer Pain Drug. National Press Office, Rockville/MD, January 16, 2008.

43. Freye E, Levy JV. Opioids in Medicine - A Comprehensive Review on the Mode of Action and the Use of Analgesics in Different Clinical Pain States. Dordrecht/NL: Springer Science + Business Media BV; 2008.

44. Mercadante S, Arcuri E, Ferrera P, Villari P, Mangione S. Alternative treatments of breakthrough pain in patients receiving spinal analgesics for cancer pain. J Pain Symptom Manage. 2005;30:485-491. 